\title{
Uniqueness and value distribution for difference operators of meromorphic function
}

\author{
Xiaoguang Qi ${ }^{1 *}$, Jia Dou ${ }^{2}$ and Lianzhong Yang ${ }^{3}$
}

\footnotetext{
* Correspondence: xiaogqi@gmail. com

${ }^{1}$ University of Jinan, School of Mathematics, Jinan, Shandong 250022, P. R. China

Full list of author information is available at the end of the article
}

\author{
Abstract \\ We investigate the value distribution of difference operator for meromorphic \\ functions. In addition, we study the sharing value problems related to a \\ meromorphic function $f(z)$ and its shift $f(z+c)$.
}

\section{Introduction and main results}

A meromorphic function means meromorphic in the whole complex plane. We assume that the reader is familiar with standard symbols and fundamental results of Nevanlinna Theory [1]. As usual, the abbreviation CM stands for "counting multiplicities", while IM means "ignoring multiplicities", and we denote the order of meromorphic function $f$ by $\sigma(f)$. For a non-constant meromorphic function $f$ and a set $S$ of complex numbers, we define the set $E(S, f)=\cup_{a \in S}\{z \mid f(z)-a=0\}$, where a zero of $f$ - $a$ with multiplicity $m$ counts $m$ times in $E(S, f)$.

We define difference operator as $\Delta_{c} f=f(z+c)-f(z)$, where $c$ is a non-zero constant. In particular, we denote by $S(f)$ the family of all meromorphic functions $a(z)$ that satisfy $T(r, a)=S(r, f)=o(T(r, f))$, where $r \rightarrow \infty$ outside a possible exceptional set of finite logarithmic measure. For convenience, we set $\hat{S}(f):=S(f) \cup\{\infty\}$.

The difference Nevanlinna theory and its applications to the uniqueness theory have become a subject of great interest [2-4], recently. With these fundamental results, Heittokangas et al. considered a meromorphic function $f(z)$ sharing values with its shift $f(z$ $+c$ ), we recall a key result from [5].

Theorem A [[5], Theorem 2]. Let $f$ be a non-constant meromorphic function of finite order, let $c \in \mathbb{C}$, and let $a, b, c \in \hat{S}(f)$ be three distinct periodic functions with period $c$. If $f(z)$ and $f(z+c)$ share $a, b C M$ and $c$ IM, then $f(z)=f(z+c)$ for all $z \in \mathbb{C}$.

Recently, Yang and Liu and one of the present authors [6] considered the case $F=f^{n}$, where $f$ is a meromorphic function, assuming value sharing with $F$ and $F(z+c)$ :

Theorem B [[6], Theorem 1.4]. Let $f$ be a non-constant meromorphic function of finite order, $n \geq 7$ be an integer, let $c \in \mathbb{C}$, and let $F=f^{n}$. If $F(z)$ and $F(z+c)$ share a $\in S(f) \backslash\{0\}$ and $\infty C M$, then $f(z)=\omega f(z+c)$, for a constant $\omega$ that satisfies $\omega^{n}=1$.

Next, we consider the problem that related to the Theorem B, and have the following result, where $a$ is a periodic function with period $c$. However, our proof is different to the one in [6].

Theorem 1.1. Let $f$ be a non-constant meromorphic function of finite order, let $c \in \mathbb{C}$, and let $a \in S(f) \backslash\{0\}$ be a periodic function with period $c$. If $f(z)^{n}$ and $f(z+c)^{n}$ share a 
and $\infty C M$, and $n \geq 4$ is an integer, then $f(z)=\omega f(z+c)$, for a constant $\omega$ that satisfies $\omega^{n}=1$.

\section{Remarks.}

(1) Theorem 1.1 is not true, if $a=0$. This can be seen by considering $f(z)=e^{z^{2}}$. Then $f(z)^{n}$ and $f(z+c)^{n}$ share 0 and $\infty \mathrm{CM}$, however, $f(z) \neq \omega f(z+c)$, where $n$ is a positive integer.

(2) Theorem 1.1 does not remain valid when $n=1$. For example, $f(z)=e^{z}+1$ and $f(z+c)=e^{z+c}+1$, where $c \neq 2 \pi i$. Clearly, $f(z)$ and $f(z+c)$ share 1 and $\infty \mathrm{CM}$, however, $f(z) \neq \omega f(z+c)$ for $\omega^{n}=1$. Unfortunately, we have not succeeded in reducing the condition $n \geq 4$ to $n \geq 2$ in Theorem 1.1, and we also cannot give a counterexample when $n=2,3$ at present.

(3) We give an example to show that the restriction of finite order in Theorem 1.1 cannot be deleted. This can be seen by taking $f(z)=e^{e^{z}}, n e^{c}=-1$. Then $f(z)^{n}$ and $f(z+c)^{n}$ share 1 and $\infty \mathrm{CM}$, however, $f(z) \neq \omega f(z+c)$, where $n$ is a positive integer.

In 1976, Gross asked the following question [[7], Question 6]:

Question. Can one find (even one set) finite sets $S_{j}(j=1,2)$ such that any two entire functions $f$ and $g$ satisfying $E\left(S_{j}, f\right)=E\left(S_{j}, g\right)(j=1,2)$ must be identical?

Since then, many results have been obtained for this and related topics (see [8-11]). We recall the following result given by Yi [9].

Theorem C [[9], Theorem 1]. Let $S_{1}=\left\{\omega \mid \omega^{n}+a \omega^{n-1}+b=0\right\}$, where $n \geq 7$ is an integer, $a$ and $b$ are two non-zero constants such that the algebraic equation $\omega^{n}+a \omega^{n-}$ ${ }^{1}+b=0$ has no multiple roots. If $f$ and $g$ are two entire functions satisfying $E\left(S_{1}, f\right)=$ $E\left(S_{1}, g\right)$, then $f=g$.

Afterwards, Fang and Lahiri [12] got the result for meromorphic functions.

Theorem D [[12], Theorem 1]. Let $S_{1}$ be defined as Theorem $C$ and $S_{2}=\{\infty\}$. Assume that $f$ and $g$ are two meromorphic functions satisfying $E\left(S_{j}, f\right)=E\left(S_{j}, g\right)$ for $j=$ 1,2. If $f$ has no simple poles and $n \geq 7$, then $f=g$.

Next, we give a difference analog of Theorem D that replacing $g$ with $f(z+c)$, and obtain the following result.

Theorem 1.2. Let $S_{1}$ be defined as Theorem $C$ and $S_{2}=\{\infty\}$. Assume that $f$ is a meromorphic function of finite order satisfying $E\left(S_{j}, f\right)=E\left(S_{j}, f(z+c)\right)$ for $j=1$,2. If $n \geq 6$ and $\bar{N}(r, f)<\frac{n-3}{n-1} T(r, f)+S(r, f)$, then $f(z)=f(z+c)$ for all $z \in \mathbb{C}$.

We investigate the value distribution of difference polynomials of meromorphic (entire) functions. Let $f$ be a transcendental meromorphic function, and let $n$ be a positive integer. Concerning to the value distribution of $f^{\imath} f^{\prime \prime}$, Hayman [[13], Corollary to Theorem 9] proved that $f^{n} f$ takes every non-zero complex value infinitely often if $n \geq$ 3. Mues [[14], Satz 3] proved that $f^{2} f-1$ has infinitely many zeros. Later on, Bergweiler and Eremenko [[15], Theorem 2] showed that ff - 1 has infinitely many zeros also. As an analog result in difference, Laine and Yang [16] investigated the value distribution of difference products of entire functions, and obtained the following: 
Theorem E [[16], Theorem 2]. Let $f$ be a transcendental entire function of finite order, and let $c$ be a non-zero complex constant. Then for $n \geq 2, f(z)^{n} f(z+c)$ assumes every non-zero value $a \in \mathbb{C}$ infinitely often.

In a recent article, one of the present authors considered the value distribution of $f$ $(z)^{n}(f(z)-1) f(z+c)$, the result may be stated as follows:

Theorem F [[17], Theorem 1]. Let $f$ be a transcendental meromorphic function of finite order $\sigma(f)$, let $a \neq 0$ be a small function with respect to $f$, and let $c$ be a non-zero complex constant. If the exponent of convergence of the poles of $f$ satisfies $\lambda\left(\frac{1}{f}\right)<\sigma(f)$ and $n \geq 2$, then $f(z)^{n}(f-1) f(z+c)$ - a has infinitely many zeros.

In this article, we replace $f(z+c)$ with $\Delta_{c} f$, and consider the value distribution of $f$ $(z)^{n}(f(z)-1) \Delta_{c} f$. We get the following results:

Theorem 1.3. Let $f$ be a transcendental meromorphic function of finite order $\sigma(f)$ and $\Delta_{c} f \neq 0$, let $a \neq 0$ be a small function with respect to $f$, and let $c$ be a non-zero complex constant. If the exponent of convergence of the poles off satisfies $\lambda\left(\frac{1}{f}\right)<\sigma(f)$ and $n \geq 3$, then $f(z)^{n}(f-1) \Delta_{c} f$ - a has infinitely many zeros.

Corollary 1.4. Let $f$ be a transcendental entire function of finite order and $\Delta_{C} f \neq 0$, let $a \neq 0$ be a small function with respect to $f$, and let $c$ be a non-zero complex constant. Then for $n \geq 3, f(z)^{n}(f-1) \Delta_{c} f$ - a has infinitely many zeros.

In particular, if $a$ is a non-zero polynomial in Corollary 1.4, then Corollary 1.4 can be improved.

Theorem 1.5. Let $f$ be a transcendental entire function of finite order and $\Delta_{c} f \neq 0$, let $a$ be a non-zero polynomial, and let $c$ be a non-zero complex constant. Then for $n \geq 2, f$ $(z)^{n}(f-1) \Delta_{f} f-a$ has infinitely many zeros.

\section{Preliminary lemmas}

Lemma 2.1. [[4], Theorem 2.1] Let $f$ be a meromorphic function of finite order, and let $c \in \mathbb{C}$ and $\delta \in(0,1)$. Then

$$
m\left(r, \frac{f(z+c)}{f(z)}\right)+m\left(r, \frac{f(z)}{f(z+c)}\right)=o\left(\frac{T(r, f)}{r^{\delta}}\right)=S(r, f) .
$$

Chiang and Feng have obtained similar estimates for the logarithmic difference [[3], Corollary 2.5], and this study is independent from [4].

Lemma 2.2. [[4], Lemma 2.3] Let $f$ be a meromorphic function of finite order and $c \in$ C. Then for any small function $a \in S(f)$ with period $c$,

$$
m\left(r, \frac{\Delta_{c} f}{f-a}\right)=S(r, f) .
$$

Lemma 2.3. [[3], Theorem 2.1] Let $f$ be a meromorphic function of finite order $\sigma(f)$, and let $c$ be a non-zero constant. Then, for each $\varepsilon>0$, we have

$$
T(r, f(z+c))=T(r, f(z))+O\left(r^{\sigma(f)-1+\varepsilon}\right)+O(\log r) .
$$

Lemma 2.4. [[18], Theorem 2.4.2] Let $f$ be a transcendental meromorphic solution of

$$
f^{n} A(z, f)=B(z, f)
$$


where $A(z, f), B(z, f)$ are differential polynomials in $f$ and its derivatives with small meromorphic coefficients $a_{\lambda}$, in the sense of $m\left(r, a_{\lambda}\right)=S(r, f)$ for all $\lambda \in I$. If the $\operatorname{deg}(B$ $(z, f)) \leq n$, then $m(r, A(z, f))=S(r, f)$.

Lemma 2.5. Let $f$ be a finite order entire function and $\Delta_{c} f \neq 0$, and let $c$ be a nonzero constant. Then

$$
m\left(r, f f^{\prime} \Delta_{c} f\right) \geq T(r, f)+S(r, f) .
$$

Proof. Since $f$ is an entire function with finite order, we deduce from Lemma 2.2 and the Lemma of logarithmic derivative that

$$
\begin{aligned}
3 T(r, f) & =T\left(r, f^{3}\right)=m\left(r, f^{3}\right)+S(r, f) \\
& \leq m\left(r, \frac{f^{3}}{f f^{\prime} \Delta_{c} f}\right)+m\left(r, f f^{\prime} \Delta_{c} f\right)+S(r, f) \\
& =m\left(r, \frac{f^{2}}{f^{\prime} \Delta_{c} f}\right)+m\left(r, f f^{\prime} \Delta_{c} f\right)+S(r, f) \\
& \leq T\left(r, \frac{f^{\prime}}{f}\right)+T\left(r, \frac{\Delta_{c} f}{f}\right)+m\left(r, f f^{\prime} \Delta_{c} f\right)+S(r, f) \\
& \leq 2 N\left(r, \frac{1}{f}\right)+m\left(r, f f^{\prime} \Delta_{c} f\right)+S(r, f) \\
& \leq 2 T(r, f)+m\left(r, f f^{\prime} \Delta_{c} f\right)+S(r, f) .
\end{aligned}
$$

Hence, we get

$$
m\left(r, f f^{\prime} \Delta_{c} f\right) \geq T(r, f)+S(r, f)
$$

\section{Proof of Theorem 1.1}

Since $f(z)^{n}$ and $f(z+c)^{n}$ share $a$ and $\infty \mathrm{CM}$, we obtain that

$$
\frac{f(z+c)^{n}-a(z+c)}{f(z)^{n}-a(z)}=e^{Q(z)},
$$

where $Q(z)$ is a polynomial. From Lemma 2.1 , we know that $T\left(r, e^{Q(z)}\right)=m\left(r, e^{Q(z)}\right)$ $=S(r, f)$. Rewrite (2) as

$$
f(z+c)^{n}=e^{Q(z)}\left(f(z)^{n}-a(z)+a(z) e^{-Q(z)}\right) .
$$

Set

$$
G(z)=\frac{f(z)^{n}}{a(z)\left(1-e^{-Q(z)}\right)} .
$$

If $e^{Q(z)} \otimes 1$, then we apply the Valiron-Mohon'ko theorem and the second main theorem to $G(z)$, and get

$$
\begin{aligned}
n T(r, f) & +S(r, f)=T(r, G) \leq \bar{N}\left(r, \frac{1}{G}\right)+\bar{N}(r, G)+\bar{N}\left(r, \frac{1}{G-1}\right)+S(r, G) \\
& \leq \bar{N}\left(r, \frac{1}{f}\right)+\bar{N}(r, f)+\bar{N}\left(r, \frac{1}{f(z)^{n}-a(z)+a(z) e^{-Q(z)}}\right)+S(r, f) \\
& \leq \bar{N}\left(r, \frac{1}{f}\right)+\bar{N}(r, f)+\bar{N}\left(r, \frac{1}{f(z+c)}\right)+S(r, f) \\
& \leq 2 T(r, f)+T(r, f(z+c))+S(r, f) .
\end{aligned}
$$


Combining (4) with Lemma 2.3, we get

$$
n T(r, f) \leq 3 T(r, f)+O\left(r^{\sigma(f)-1+\varepsilon}\right)+S(r, f)
$$

which contradicts that $n \geq 4$. Therefore, $e^{Q(z)} \equiv 1$, that is, $f(z)^{n}=f(z+c)^{n}$, so we have $f(z)=\omega f(z+c)$, for a constant $\omega$ with $\omega^{n}=1$.

\section{Proof of Theorem $\mathbf{1 . 2}$}

From the assumption of Theorem 1.2, we get that

$$
\frac{f(z+c)^{n}+a f(z+c)^{n-1}+b}{f(z)^{n}+a f(z)^{n-1}+b}=e^{Q(z)},
$$

where $Q(z)$ is a polynomial. Applying Lemma 2.1, we obtain that $T\left(r, e^{Q(z)}\right)=m(r$, $\left.e^{Q(z)}\right)=S(r, f)$. Rewrite (5) as

$$
f(z+c)^{n}+a f(z+c)^{n-1}=e^{Q(z)}\left(f(z)^{n}+a f(z)^{n-1}+b-\frac{b}{e^{Q(z)}}\right) .
$$

If $e^{Q(z)} \otimes 1$, applying the second main theorem for three small functions, we get

$$
\begin{aligned}
n T(r, f)+S(r, f) & =T\left(r, f(z)^{n}+a f(z)^{n-1}\right) \\
& \leq \bar{N}\left(r, \frac{1}{f(z)^{n}+a f(z)^{n-1}}\right)+\bar{N}\left(r, f(z)^{n}+a f(z)^{n-1}\right) \\
& +\bar{N}\left(r, \frac{1}{f(z)^{n}+a f(z)^{n-1}+b-\frac{b}{e^{Q(z)}}}\right)+S(r, f) \\
& \leq \bar{N}(r, f)+\bar{N}\left(r, \frac{1}{f(z+c)^{n-1}(f(z+c)+a)}\right) \\
& +\bar{N}\left(r, \frac{1}{f(z)^{n-1}(f(z)+a)}\right)+S(r, f) \\
& \leq 3 T(r, f)+2 T(r, f(z+c))+S(r, f) .
\end{aligned}
$$

Combining (4.3) with Lemma 2.3, we get

$$
n T(r, f) \leq 5 T(r, f)+O\left(r^{\sigma(f)-1+\varepsilon}\right)+S(r, f)
$$

which contradicts $n \geq 6$. Hence, $e^{Q(z)} \equiv 1$, we conclude by (5) that

$$
f(z+c)^{n}+a f(z+c)^{n-1}=f(z)^{n}+a f(z)^{n-1} .
$$

Set $G(z)=\frac{f(z)}{f(z+c)}$. If $G(z)$ is non-constant, then we have from (8)

$$
f(z)=-\frac{a G\left(G^{n-1}-1\right)}{G^{n}-1}=-a \frac{G^{n-1}+\cdots+G}{G^{n-1}+\cdots+1} .
$$

Making use of the standard Valiron-Mohon'ko lemma, we get from (9) that

$$
T(r, f)=(n-1) T(r, G)+S(r, f) .
$$

Noting that $n \geq 6$, we deduce that 1 is not a Picard value of $G^{n}$. Suppose that $a_{j} \in\{\mathbb{C}$ $\backslash 1\}(j=1,2, \ldots, n-1)$ are the distinct roots of equation $h^{n}-1=0$. Applying the second main theorem to $G$, we conclude by (9) that 


$$
(n-3) T(r, G) \leq \sum_{j=1}^{n-1} \bar{N}\left(r, \frac{1}{G-a_{j}}\right)+S(r, G)=\bar{N}(r, f) .
$$

From (10) and (11), we get $\bar{N}(r, f) \geq \frac{n-3}{n-1} T(r, f)+S(r, f)$, which contradicts the assumption.

So $G(z)$ is a constant, and we get $f(z)=t f(z+c)$, where $t$ is a non-zero constant. From (8), we know $t=1$, therefore, $f=g$.

\section{Proof of Theorem 1.3}

The main idea of this proof is from [[17], Theorem 1], while the details are somewhat different. For the convenience of the reader, we give a complete proof.

Set $F(z)=f^{r}(z)(f(z)-1) \Delta_{c} f$. Since $f$ is a transcendental meromorphic function with finite order $\sigma(f)$, we conclude by Lemma 2.3 that

$$
\begin{aligned}
T(r, F) & \leq T\left(r, f^{n}(z)(f(z)-1)\right)+T\left(r, \Delta_{c} f\right)+S(r, f) \\
& \leq(n+2) T(r, f)+T(r, f(z+c))+S(r, f) \\
& \leq(n+3) T(r, f)+O\left(r^{\sigma(f)-1+\varepsilon}\right)+S(r, f) .
\end{aligned}
$$

Thus, we get $S(r, F)=o(T(r, f))=S(r, f)$. Moreover, we get

$$
\begin{aligned}
T\left(r, \Delta_{c} f\right) & \leq m\left(r, \Delta_{c} f\right)+O\left(r^{\lambda\left(\frac{1}{f}\right)+\varepsilon}\right)+S(r, f) \\
& \leq m\left(r, \frac{\Delta_{c} f}{f}\right)+m(r, f)+O\left(r^{\lambda\left(\frac{1}{f}\right)+\varepsilon}\right)+S(r, f) \\
& \leq T(r, f)+O\left(r^{\lambda\left(\frac{1}{f}\right)+\varepsilon}\right)+S(r, f) .
\end{aligned}
$$

On the other hand, we deduce by Lemma 2.2 that

$$
\begin{aligned}
(n+2) T(r, f) & =T\left(r, f^{n+1}(f-1)\right)+S(r, f) \\
& =m\left(r, f^{n+1}(f-1)\right)+O\left(r^{\lambda\left(\frac{1}{f}\right)+\varepsilon}\right)+S(r, f) \\
& \leq m\left(r, \frac{f^{n+1}(f-1)}{F}\right)+m(r, F)+O\left(r^{\lambda\left(\frac{1}{f}\right)+\varepsilon}\right)+S(r, f) \\
& \leq T\left(r, \frac{\Delta_{c} f}{f}\right)+m(r, F)+O\left(r^{\lambda\left(\frac{1}{f}\right)+\varepsilon}\right)+S(r, f) \\
& \leq m\left(r, \frac{\Delta_{c} f}{f}\right)+N\left(r, \frac{1}{f}\right)+m(r, F)+O\left(r^{\lambda\left(\frac{1}{f}\right)+\varepsilon}\right)+S(r, f) \\
& \leq T(r, f)+T(r, F)+O\left(r^{\lambda\left(\frac{1}{f}\right)+\varepsilon}\right)+S(r, f) .
\end{aligned}
$$

Hence

$$
(n+1) T(r, f) \leq T(r, F)+O\left(r^{\lambda\left(\frac{1}{f}\right)+\varepsilon}\right)+S(r, f) .
$$


The second main theorem yields

$$
\begin{aligned}
T(r, F) \leq & \bar{N}(r, F)+\bar{N}\left(r, \frac{1}{F}\right)+\bar{N}\left(r, \frac{1}{F-a}\right)+S(r, F) \\
\leq & \bar{N}\left(r, \frac{1}{F-a}\right)+\bar{N}\left(r, \frac{1}{f}\right)+\bar{N}\left(r, \frac{1}{f-1}\right) \\
& +\bar{N}\left(r, \frac{1}{\Delta_{c} f}\right)+O\left(r^{\lambda\left(\frac{1}{f}\right)+\varepsilon}\right)+S(r, f) \\
\leq & \bar{N}\left(r, \frac{1}{F-a}\right)+2 T(r, f)+T\left(r, \Delta_{c} f\right)+O\left(r^{\lambda\left(\frac{1}{f}\right)+\varepsilon}\right)+S(r, f) .
\end{aligned}
$$

From (12) and above inequality, we get that

$$
T(r, F) \leq \bar{N}\left(r, \frac{1}{F-a}\right)+3 T(r, f)+O\left(r^{\lambda\left(\frac{1}{f}\right)+\varepsilon}\right)+S(r, f) .
$$

Combining (13) and (14), we have

$$
(n-2) T(r, f) \leq \bar{N}\left(r, \frac{1}{F-a}\right)+O\left(r^{\lambda\left(\frac{1}{f}\right)+\varepsilon}\right)+S(r, f),
$$

which is a contradiction to the fact that $f$ is of order $\sigma(f)$ if $F$ - $a$ has finitely many zeros. The conclusion follows.

\section{Proof of Theorem 1.5}

Suppose that $f^{n}(f-1) \Delta_{c} f$ - $a$ admits finitely many zeros only. Then, there are two nonzero polynomials $P(z), Q(z)$ such that

$$
f^{n}(f-1) \Delta_{c} f-a=P(z) e^{Q(z)} .
$$

Differentiating (15) and eliminating $e^{Q(z)}$, we obtain

$$
\left(f^{n}-f^{n-1}\right) F(z, f)=a^{\prime} P(z)-a P^{*}(z)-P(z) f(z)^{n-1} f^{\prime}(z) \Delta_{c} f,
$$

where

$$
F(z, f)=(n+1) P(z) f^{\prime}(z) \Delta_{c} f+P(z) f(z)\left(\Delta_{c} f\right)^{\prime}-P^{*}(z) f(z) \Delta_{c} f
$$

and $P^{*}(z)=P^{\prime}(z)+P(z) Q^{\prime}(z)$.

First, we conclude that $a^{\prime} P(z)-a P^{*}(z) \otimes 0$. Otherwise, if $a^{\prime} P(z)-a P^{*}(z)=0$, by integrating, then we have

$$
\frac{a}{P(z)}=A e^{Q(z)}
$$

where $A$ is a non-zero constant. Hence, we get $e^{Q(z)}$ is a constant and

$$
f^{n}(z)(f(z)-1) \Delta_{c} f=B P(z)+a,
$$

where $B$ is a non-zero constant. Then, from Lemma 2.3 and (17), we obtain that

$$
(n+1) T(r, f) \leq 2 T(r, f)+O\left(r^{\sigma(f)-1+\varepsilon}\right)+S(r, f),
$$

which is a contradiction when $n \geq 2$. 
If $F(z, f)$ vanish identically, then

$$
a P^{*}(z)+P(z) f(z)^{n-1} f^{\prime}(z) \Delta_{c} f-a^{\prime} P(z) \equiv 0 .
$$

Rewrite (18), we get

$$
f^{n-2} f f^{\prime}(z) \Delta_{c} f=\frac{a^{\prime} P(z)-a P^{*}(z)}{P(z)},
$$

hence

$$
f^{n-2} f^{2} f^{\prime}(z) \frac{\Delta_{c} f}{f}=\frac{a^{\prime} P(z)-a P^{*}(z)}{P(z)} .
$$

Then, combining Lemmas 2.2, 2.4 and Equation (19), we conclude that

$$
m\left(r, f f^{\prime}(z) \Delta_{c} f\right)=S(r, f),
$$

which contradicts (1).

It remains to consider the case that $F(z, f) \otimes 0$. We rewrite (16) in the form that

$$
\left(f(z)^{n+2}-f(z)^{n+1}\right) \frac{F(z, f)}{f(z)^{2}}=a^{\prime} P(z)-a P^{*}(z)-P(z) f(z)^{n-1} f^{\prime}(z) \Delta_{c} f
$$

and

$$
f(z)^{n+1}\left((f(z)-1) \frac{F(z, f)}{f(z)^{2}}\right)=a^{\prime} P(z)-a P^{*}(z)-P(z) f(z)^{n-1} \frac{f^{\prime}(z) \Delta_{c} f}{f(z)^{2}} .
$$

By Lemmas 2.2 and 2.4, we know that

$$
m\left(r, \frac{F(z, f)}{f(z)^{2}}\right)=S(r, f)
$$

and

$$
m\left(r,(f(z)-1) \frac{F(z, f)}{f(z)^{2}}\right)=S(r, f) .
$$

As $f(z)$ is entire, we get that the poles of $\frac{F(z, f)}{f(z)^{2}}$ may be located only at the zeros of $f$ $(z)$. If $\frac{F(z, f)}{f(z)^{2}}$ has infinitely many poles, then from that a zero of $f(z)$ with multiplicity $t$ should be a pole of $t+1$ of $\frac{F(z, f)}{f(z)^{2}}$. Since $n \geq 2$, we know that the left side of (20) must have infinitely many zeros, which is a contradiction that $a^{\prime} P(z)-a P^{* *}(z)$ is a non-zero polynomial. We get

$$
N\left(r, \frac{F(z, f)}{f(z)^{2}}\right)=O(\log r) \quad \text { and } \quad N\left(r,(f(z)-1) \frac{F(z, f)}{f(z)^{2}}\right)=O(\log r) .
$$

Hence

$$
T\left(r, \frac{F(z, f)}{f(z)^{2}}\right)=S(r, f)
$$


and

$$
T\left(r,(f(z)-1) \frac{F(z, f)}{f(z)^{2}}\right)=S(r, f)
$$

as well. Combining these two estimates, we obtain

$$
T(r, f)=S(r, f)
$$

\section{contradiction. This completes the proof of Theorem 1.5.}

\section{Acknowledgements}

The authors thank the referee for his/her valuable suggestions to improve the present article. This study was supported by the NSF of Shandong Province, China (No. ZR2010AM030).

\section{Author details}

${ }^{1}$ University of Jinan, School of Mathematics, Jinan, Shandong 250022, P. R. China ${ }^{2}$ Quancheng Middle School, Jinan, Shandong 250000, P. R. China ${ }^{3}$ Shandong University, School of Mathematics, Jinan, Shandong 250100, P. R. China

\section{Authors' contributions}

XQ completed the main part of this article, JD and LY corrected the main theorems. All authors read and approved the final manuscript.

\section{Competing interests}

The authors declare that they have no competing interests.

\section{Received: 11 August 2011 Accepted: 14 March 2012 Published: 14 March 2012}

\section{References}

1. Yang, CC, Yi, HX: Uniqueness Theory of Meromorphic Functions. Kluwer Academic Publishers, Dordrecht (2003)

2. Bergweiler, W, Langley, JK: Zeros of difference of meromorphic functions. Math Proc Cambridge Philos Soc. 142, 133-147 (2007). doi:10.1017/S0305004106009777

3. Chiang, YM, Feng, SJ: On the Nevanlinna characteristic of $f(z+\eta)$ and difference equations in the complex plane. Ramanujan J. 16, 105-129 (2008). doi:10.1007/s11139-007-9101-1

4. Halburd, RG, Korhonen, RJ: Nevanlinna theory for the difference operator. Ann Acad Sci Fenn. 31, 463-478 (2006)

5. Heittokangas, J, Korhonen, R, Laine, I, Rieppo, J, Zhang, JL: Value sharing results for shifts of meromorphic function, and sufficient conditions for periodicity. J Math Anal Appl. 355, 352-363 (2009). doi:10.1016/j.jmaa.2009.01.053

6. Qi, XG, Yang, LZ, Liu, K: Uniqueness and periodicity of meromorphic functions concerning difference operator. Comput Math Appl. 60, 1739-1746 (2010). doi:10.1016/j.camwa.2010.07.004

7. Gross, F: Factorization of meromorphic functions and some open problems, Complex analysis (Proc. Conf., Univ. Kentucky, Lexington, Ky., 1976). In Lecture Notes in Math, vol. 599, pp. 51-67.Springer, Berlin (1977). doi:10.1007/ BFb0096825

8. Frank, G, Reinders, M: A unique range set for meromorphic functions with 11 elements. Complex var Theory Appl. 37, 185-193 (1998). doi:10.1080/17476939808815132

9. Yi, HX: A question of Gross and the uniqueness of entire functions. Nagoya Math J. 138, 169-177 (1995)

10. Yi, HX: Unicity theorems for meromorphic or entire functions II. Bull Austral Math Soc. 52, 215-224 (1995). doi:10.1017/ S0004972700014635

11. Yi, HX: Unicity theorems for meromorphic or entire functions III. Bull Austral Math Soc. 53, 71-82 (1996). doi:10.1017/ S0004972700016737

12. Fang, ML, Lahiri, I: Unique range set for certain meromorphic functions. Indian J Math. 45, 141-150 (2003)

13. Hayman, WK: Picard values of meromorphic functions and their derivatives. Ann Math. 70(2):9-42 (1959)

14. Mues, E: Über ein Problem von Hayman. Math Z. 164, 239-259 (1979). doi:10.1007/BF01182271

15. Bergweiler, W, Eremenko, A: On the singularities of the inverse to a meromorphic function of finite order. Rev Mat Iberoamericana. 11, 355-373 (1995)

16. Laine, I, Yang, CC: Value Distribution of Difference Polynomials. Proc Japan Acad Ser A Math Sci. 83, 148-151 (2007). doi:10.3792/pjaa.83.148

17. Qi, XG: Value distribution and uniqueness of difference polynomials and entire solutions of difference equations. Ann Polon Math. 102(2):129-142 (2011). doi:10.4064/ap102-2-3

18. Laine, I: Nevanlinna Theory and Complex Differential Equations. Walter de Gruyter. Berlin-New York (1993)

doi:10.1186/1687-1847-2012-32

Cite this article as: Qi et al:: Uniqueness and value distribution for difference operators of meromorphic function. Advances in Difference Equations 2012 2012:32. 